\title{
Reusing Decisions Made with One Decision Support System to Assess a Second Decision Support System: Introducing the Notion of Complex Cases
}

\author{
Akram REDJDAL ${ }^{\mathrm{a}, 1}$, Jacques BOUAUD ${ }^{\mathrm{b}, \mathrm{a}}$, Gilles GUÉZENNEC ${ }^{\mathrm{a}}$, Joseph \\ GLIGOROV $^{\mathrm{c}, \mathrm{d}}$ and Brigitte SEROUSSI ${ }^{\mathrm{a}, \mathrm{d}}$ \\ a Sorbonne Université, Université Sorbonne Paris Nord, Inserm, \\ UMRS 1142, LIMICS, Paris, France \\ ${ }^{\mathrm{b}}$ AP-HP, DRCI, Paris, France
}

'Sorbonne Université, Institut Universitaire de Cancérologie, Paris, France

d AP-HP, Hôpital Tenon, Paris, France

\begin{abstract}
The guideline-based decision support system (GL-DSS) of the DESIREE project and OncoDoc are two clinical decision support systems applied to the management of breast cancer. In order to evaluate the DESIREE GL-DSS, we decided to reuse a sample of clinical cases previously resolved by the multidisciplinary tumor board (MTB) of the Tenon Hospital (Paris, France) when using OncoDoc. Since we had two different knowledge representation models to represent clinical parameters and decisions, and two formalisms to represent guidelines, we developed a transformation sequence, involving the creation of synthetic patients, the enrichment of DESIREE ontology, and the translation of clinical cases and their decisions, to transform OncoDoc data into the DESIREE representation. Considering MTB decisions as the gold standard, the $84 \%$ compliance rate of DESIREE recommendations was rather satisfactory. Some situations $(0.7 \%)$ concerned clinical cases that were compliant neither with OncoDoc nor with DESIREE that we defined as complex cases, not handled by guidelines, which necessitate effective MTB discussions.
\end{abstract}

Keywords. Clinical decision support systems, Clinical practice guidelines, Ontologies, Interoperability, Evaluation, Breast cancer

\section{Introduction}

Clinical decision support systems (CDSSs) are software components that aim to support clinicians in their decision-making process. CDSSs have been used in many medical areas. They have proven to improve the quality of patient care by increasing the compliance of clinicians' decisions with clinical practice guidelines (CPGs) [1].

DESIREE $^{2}$ is a recent European-funded project which aimed at developing a webbased platform to improve the management of primary breast cancer patients. Among

\footnotetext{
${ }^{1}$ Corresponding Author: Akram Redjdal, LIMICS UMRS_1142, 15 rue de l'Ecole de Médecine, Paris, France; E-mail: redjdalakram300@gmail.com

${ }^{2}$ The DESIREE project has received funding from the European Union's Horizon 2020 research and innovation programme under grant agreement No 690238.
} 
other services, DESIREE includes a guideline-based decision support system (GL-DSS) that some authors of this article have developed [2]. As part of the DESIREE project, we had to evaluate the GL-DSS. However, we were lacking a large sample of resolved clinical cases, including breast cancer patient data and their corresponding decisions. Since some authors of this article had previously developed OncoDoc [3], another guideline-based CDSS for the management of primary breast cancer patients, and since OncoDoc had been routinely used by the multidisciplinary tumor board (MTB) of the Tenon hospital (Paris, France) for three years, we decided to reuse the set of 1,861 MTB decisions made with OncoDoc to assess the DESIREE GL-DSS.

As the two CDSSs make use of two different domain knowledge models and two different formalisms to represent breast cancer data and CPGs, we developed and implemented a model transformation from OncoDoc to DESIREE to be able to evaluate the GL-DSS with data generated from OncoDoc. Because one-to-one matching between the domain knowledge concepts of OncoDoc and DESIREE could not systematically be performed, we created "synthetic" patients to translate OncoDoc clinical cases in the DESIREE formalism [4]. Going further in the evaluation of the DESIREE GL-DSS, the aim of this paper is to describe the enrichment of the DESIREE ontology to integrate OncoDoc concepts, how the inference engine of the GL-DSS was used to implement the model transformation, and the results of the evaluation of the DESIREE GL-DSS.

\section{Material and Methods}

\subsection{Creation of OncoDoc-derived synthetic patients}

OncoDoc relies on a knowledge base modeling Cancer-Est CPGs. The knowledge base is structured as a decision tree that the user navigates through while interactively answering questions to instantiate patient parameters [3]. Nodes represent decision variables and edges represent their modalities. Data representation in DESIREE relies on a Breast Cancer Knowledge Model (BCKM) formalized as an ontology built from CPG features [5] and enriched by concepts extracted from the NCI thesaurus. The BCKM allows for rule-based and subsumption-based reasoning to provide best patientcentered guideline-based recommendations. The BCKM relies on a data model based on the generic Entity-Attribute-Value (EAV) model [2] (see Figure 1), the main entities being the patient, the breast side, and the lesion. CPGs used in the project, including the APHP CPGs considered as the update of CancerEst CPGs, are formalized as sets of Natural Rule Language (NRL) rules [6] matching the data model described in the BCKM.

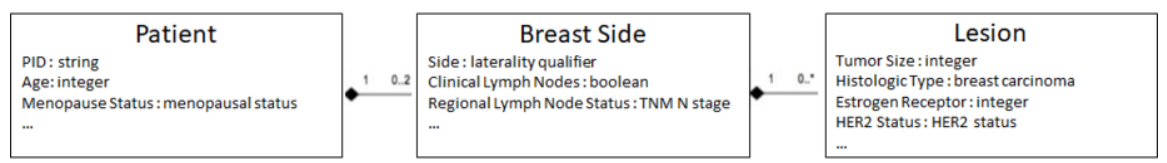

Figure 1. EAV model in DESIREE

We have used the sample of clinical cases previously resolved with OncoDoc by the MTBs of the Tenon Hospital [3]. Each case includes the description of the patient profile as a set of instantiated clinical parameters and the corresponding MTB decision.

The two CDSSs use different representations of the breast cancer domain knowledge. For example, the tumor size is represented as a categorical variable ("less 
than $2 \mathrm{~cm}$ ", "between 2 and $4 \mathrm{~cm}$ ", "more than $4 \mathrm{~cm}$ ") in OncoDoc, whereas it is represented in DESIREE as a quantitative variable in millimeters, partly related to the $\mathrm{T}$ of the TNM classification, another categorical variable. Thus, a tumor size of "more than $4 \mathrm{~cm}$ " would have two possible correspondences, T2 (more than $4 \mathrm{~cm}$ but less than $5 \mathrm{~cm}$ ) and T3 (more than $5 \mathrm{~cm}$ ). To solve this interoperability issue, for each OncoDoc patient, we generated potential corresponding DESIREE synthetic patients [4].

\subsection{Running the DESIREE GL-DSS on OncoDoc-derived synthetic patients}

The aim is to get DESIREE's recommendations for each synthetic patient and compare these recommendations with the decision made for its originating OncoDoc patient. For this, we developed a model transformation made of five steps as displayed in Figure 2.

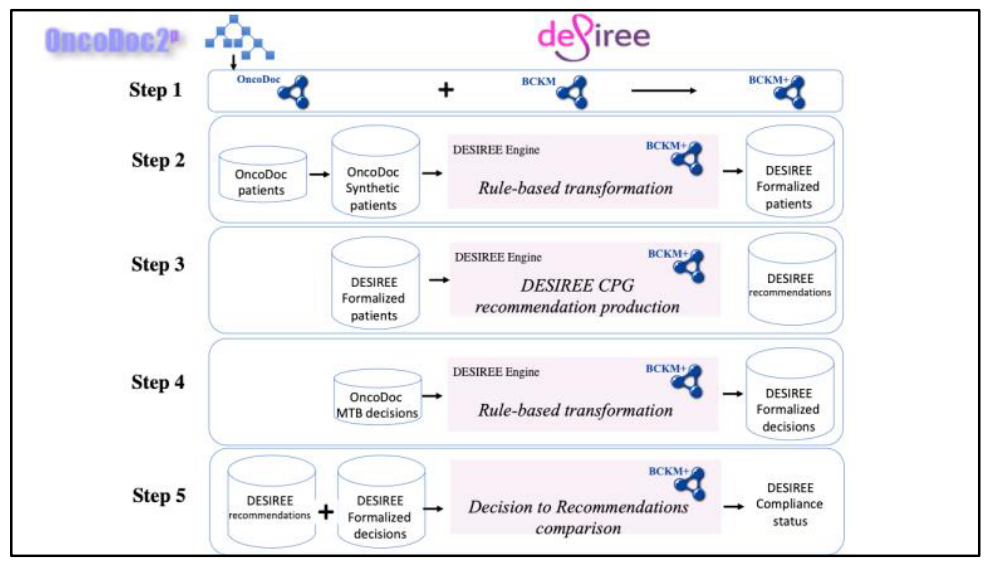

Figure 2. Model transformation from OncoDoc to DESIREE

Step 1: Enrichment of the BCKM ontology with OncoDoc concepts. We started by creating a new ontology based on the same EAV model than the DESIREE BCKM to represent Oncodoc parameters. In this new ontology, we have one entity, the OncoDoc patient entity which has OncoDoc attributes to represent the clinical parameters with values that may be either boolean (true or false), or categorical (e.g., the tumor size). The new ontology was then included within the BCKM to get a comprehensive ontology, denoted BCKM+, embedding concepts of both OncoDoc and DESIREE.

Step 2: Translation of OncoDoc-derived synthetic patients into DESIREE-formalized patients. We built NRL IF-THEN rules to align the concepts within the BCKM+. These rules allowed to create for each synthetic patient (i) a new patient entity, side entity, and lesion entity (e.g., IF there is an OncoDocPatientEntity, THEN create a PatientEntity, AND a SideEntity AND a LesionEntity), (ii) to instantiate all attributes by mapping OncoDoc concepts to DESIREE concepts within the BCKM+, and (iii) to translate OncoDoc-derived synthetic patients into DESIREE-formalized patients in compliance with BCKM formalism and semantics (e.g., IF OncoDocPatientEntity.TypeLesion = Invasive, THEN LesionEntity.HistologicType = InvasifBreastCarcinoma).

Step 3: Running DESIREE GL-DSS on DESIREE-formalized patients to get DESIREE recommendations. We re-used the pipeline of the DESIREE project.

Step 4: Translation of decisions made with OncoDoc into DESIREE-formalized decisions. We built NRL rules to translate MTB decisions made with OncoDoc into a 
DESIREE-formalized MTB decision (e.g., IF OncoDocPatientEntity.Decision = "Mastectomie" THEN Create an OrderEntity with Mastectomy using "SHOULD").

Step 5: Comparison of DESIREE-formalized decisions and DESIREE recommendations. For each patient, we compared the MTB decision and the recommendations of the GLDSS, both being represented in the same format and using the same concepts.

\subsection{Evaluation of the DESIREE GL-DSS}

We computed the compliance rate of DESIREE-formalized MTB decisions with DESIREE recommendations, making the difference between decisions that were compliant with OncoDoc and those which were not. We also analyzed the clinical cases leading to the silence of the DESIREE GL-DSS (i.e. no recommendation was issued).

\section{Results}

\subsection{Enrichment of the BCKM ontology}

The enrichment of the BCKM led to the addition of 77 OncoDoc concepts, all made of decision variables. Figure 3 displays an excerpt of the enriched ontology $\mathrm{BCKM}+\mathrm{We}$ created 347 "IF-THEN" rules to handle the translation of parameters (step 2, 173 rules) and decisions (step 3, 274 rules).

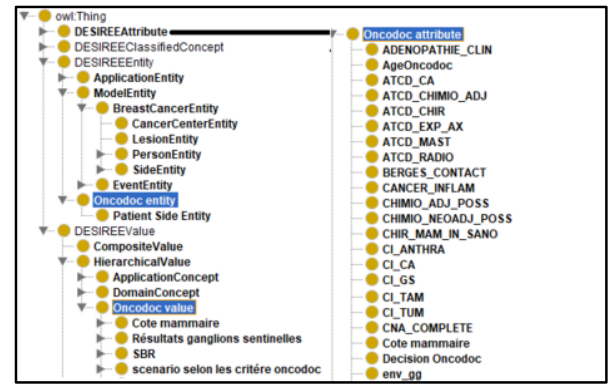

Figure 3. Overview of the new BCKM + OncoDoc ontology

\subsection{Evaluation of the DESIREE GL-DSS}

From the sample of 1,861 MTB decisions made with OncoDoc, we derived a set of 12,542 synthetic patients (Table 1). The compliance rate of MTB decisions with DESIREE was measured at $84 \%(10,481 / 12,542)$. A disagreement between OncoDoc and DESIREE was observed in $18 \%$ of the cases $(797+1,427=2,224$ decisions $)$. We noticed that 92 decisions $(0.7 \%)$ were non-compliant with both OncoDoc and DESIREE. DESIREE did not generate recommendations in $4 \%$ of the decisions $(542 / 12,542)$.

Table 1. Compliance of MTB decisions with both OncoDoc and DESIREE

\begin{tabular}{lccc}
\hline & Compliance with OncoDoc & Non-compliance with OncoDoc & Total \\
\hline Compliance with DESIREE & 9,684 & 797 & 10,481 \\
Non-compliance with DESIREE & 1,427 & 92 & 1,519 \\
Silence & 471 & 71 & 542 \\
Total & 11,582 & 960 & 12,542 \\
\hline
\end{tabular}




\section{Discussion and conclusion}

We developed a transformation suite, involving the creation of synthetic patients, the enrichment of DESIREE ontology and the translation of clinical cases and MTB decisions, to transform OncoDoc materials into DESIREE formalism and semantics. Thus, we were able to reuse past breast cancer clinical cases and their decisions made with OncoDoc to perform a first evaluation of the GL-DSS developed as part of the DESIREE project. Overall, considering MTB decisions as the gold standard, the $84 \%$ compliance rate of DESIREE recommendations was rather satisfactory. This is consistent with the fact that CDSSs have the potential to improve compliance of clinical decisions with CPGs, even if CDSS use in decision-making is still understudied [7].

The 797 decisions where DESIREE was compliant whereas OncoDoc was noncompliant can be explained by the evolution of practices and the improvement of CPGs to cover more clinical situations. We analyzed the special case of the 1,427 decisions that were compliant with OncoDoc and non-compliant with DESIREE, and observed that the created synthetic patients were too different from the original OncoDoc clinical cases to receive the same therapeutic options, e.g., when the parameter Plus $2 \mathrm{~N}$ is false in OncoDoc, two synthetic patients are created, with N0 and N1 in the TNM classification, but whereas the sentinel axillary lymph node biopsy is recommended with N0, it is prohibited with N1. In the same way, the DESIREE GL-DSS silence could be explained by clinical cases which transformation resulted in missing tumor size (Tx tumor, microinvasive cancer, or multifocal lesion). Whereas OncoDoc had special concepts to cover these situations, DESIREE had to manage the issue of incompletely specified clinical cases. Finally, the 92 decisions $(0.7 \%)$ that were non-compliant with both OncoDoc and DESIREE are a marker of "complex cases", uncovered by CPGs, which actually necessitate a thorough tumor board discussion.

\section{References}

[1] Kwan JL, Lo L, Ferguson J, et al. Computerised clinical decision support systems and absolute improvements in care: meta-analysis of controlled clinical trials. BMJ. 2020;370:3216.

[2] Bouaud J, Pelayo S, Lamy JB, et al. Implementation of an ontological reasoning to support the guidelinebased management of primary breast cancer patients in the DESIREE project. Artif Intell Med. 2020 Aug; 108:101922.

[3] Séroussi B., Bouaud J., Éric Charles Antoine OncoDoc: A successful experiment of computer-supported guideline development and implementation in the treatment of breast cancer. Artif. Intell. Med. 2001;22:43-64.

[4] Redjdal A, Bouaud J, Guézennec G, Gligorov J, Seroussi B. Creating Synthetic Patients to Address Interoperability Issues: A Case Study with the Management of Breast Cancer Patients. Stud Health Technol Inform. 2020 Nov 23;275:177-181.

[5] Quesada-Martínez M, Marcos M, Abad-Navarro F, Martínez-Salvador B, Fernández-Breis JT. Towards the semantic enrichment of Computer Interpretable Guidelines: a method for the identification of relevant ontological terms. AMIA Annu Symp Proc. 2018 Dec 5;2018:922-31.

[6] Nentwich C, James R. Natural rule language (NRL) specification 1.4.0. 2010. Available at: http://nrl.sourceforge.net/spec/. Accessed March 3, 2021.

[7] Beauchemin M, Murray MT, Sung L, Hershman DL, Weng C, Schnall R. Clinical decision support for therapeutic decision-making in cancer: A systematic review. Int J Med Inform. 2019;130:103940. 